\title{
Existence and Uniqueness of Solutions to Impulsive Fractional Integro-Differential Equations with Nonlocal Conditions
}

\author{
Zhenghui Gao, Liu Yang, Gang Liu \\ Department of Mathematics and Computational Science, Hengyang Normal University, Hengyang, China \\ Email: gzh1234567890@126.com
}

Received March 22, 2013; revised April 20, 2013; accepted April 28, 2013

Copyright (c) 2013 Zhenghui Gao et al. This is an open access article distributed under the Creative Commons Attribution License, which permits unrestricted use, distribution, and reproduction in any medium, provided the original work is properly cited.

\begin{abstract}
In this article, by using Schaefer fixed point theorem, we establish sufficient conditions for the existence and uniqueness of solutions for a class of impulsive integro-differential equations with nonlocal conditions involving the Caputo fractional derivative.
\end{abstract}

Keywords: Caputo Fractional Derivative; Impulses; Nonlocal Conditions; Existence; Uniqueness; Fixed Point

\section{Introduction}

Fractional differential equations appear naturally in a number of fields such as physics, engineering, biophysics, blood flow phenomena, aerodynamics, electron-analytical chemistry, biology, control theory, etc., An excellent account in the study of fractional differential equations can be found in [1-11] and references therein. Undergoing abrupt changes at certain moment of times like earthquake, harvesting, shock etc, these perturbations can be well-approximated as instantaneous change of state or impulses. Furthermore, these processes are modeled by impulsive differential equations. In 1960, Milman and Myshkis introduced impulsive differential equations in their papers [12]. Based on their work, several monographs have been published by many authors like Semoilenko and Perestyuk [13], Lak-shmikantham et al. [14], Bainov and Semoinov [15,16], Bainov and Covachev [17] and Benchohra et al. [18]. Impulsive fractional differential equations represent a real framework for mathematical modelling to real world problems. Significant progress has been made in the theory of impulsive fractional differential equations [19-21].

We consider a class of impulsive fractional integrodifferential equations with nonlocal conditions of the form

$$
\begin{aligned}
& { }^{c} D^{\alpha} y(t)=f\left(t, y(t), \int_{0}^{t} h(t, r) y(r) \mathrm{d} r\right), \\
& t \in J=[0, T], t \neq t_{k}, k=1,2, \cdots, m,
\end{aligned}
$$

$$
\begin{array}{r}
\left.\Delta y(t)\right|_{t=t_{k}}=I_{k}\left(y\left(t_{k}^{-}\right)\right), \\
y(0)+g(y(t))=y_{0} .
\end{array}
$$

Where ${ }^{c} D^{\alpha}$ is the Caputo fractional derivative, the function $f(t, \cdot, \cdot): J \times R^{2} \rightarrow R$ is continuous and the function $h(t, r): D \rightarrow R, D=\{(t, r) \in J \times J: 0 \leq r \leq t \leq T\}$ is continuous, $h_{0}=\max \{h(t, r):(t, r) \in D\}$;

$$
\begin{aligned}
& I_{k}: R \rightarrow R, 0=t_{0}<t_{1}<\cdots<t_{m}<t_{m+1}=T, \\
& \left.\Delta y(t)\right|_{t=t_{k}}=y\left(t_{k}^{+}\right)-y\left(t_{k}^{-}\right), \\
& y\left(t_{k}^{+}\right)=\lim _{h \rightarrow 0^{+}} y\left(t_{k}+h\right)
\end{aligned}
$$

and $y\left(t_{k}^{-}\right)=\lim _{h \rightarrow 0^{-}} y\left(t_{k}+h\right)$ represent the right and left limits of $y(t)$ at $t_{k}$, and $g: P C(J, R) \rightarrow R$ is a continuous function, $y_{0} \in R$.

Nonlocal conditions were initiated by Byszewski [22] who proved the existence and uniqueness of mild and classical solutions of nonlocal Cauchy problems. As remarked by Byszewski $[23,24]$, the nonlocal condition can be more useful than the standard initial condition to describe some physical phenomena. For example, $g(y(t))$ may be given by

$$
g(y(t))=\sum_{i=1}^{p} c_{i} y\left(\tau_{i}\right)
$$

where $c_{i}, i=1,2, \cdots, p$ are given constants and 
$0<\tau_{1}<\tau_{2}<\cdots<\tau_{p}<T$.

In this article, our aim is to show sufficient conditions for the existence and uniqueness of solutions of solutions to impulsive fractional integro-differential equations with nonlocal conditions.

\section{Preliminaries}

In this section, we introduce some notations, definitions and preliminary facts which are used throughout this paper. By $C(J, R)$ we denote the Banach space of all continuous functions from $J$ into $R$ with the norm

$$
\|y\|=\sup \{|y(t)|: t \in J\} .
$$

Definition 2.1 [5,8]: The fractional (arbitrary) order integral of the function $h \in L^{1}\left([a, b], R_{+}\right)$of order $\alpha \in R_{+}=[0,+\infty)$ is defined by

$$
I_{a}^{\alpha} h(t)=\frac{1}{\Gamma(\alpha)} \int_{a}^{t}(t-s)^{\alpha-1} h(s) \mathrm{d} s,
$$

where $\Gamma$ is the gamma function, when $a=0, I_{a}^{\alpha} h(t)=I^{\alpha} h(t)$.

Definition 2.2 [5,8]: For a function $h$ given on the interval $[a, b]$, Riemann-Liouville fractional-order derivative of order $\alpha$ of $h$, is defined by

$$
D_{a}^{\alpha} h(t)=\frac{1}{\Gamma(n-\alpha)}\left(\frac{\mathrm{d}}{\mathrm{d} t}\right)^{n} \int_{a}^{t}(t-s)^{n-\alpha-1} h(s) \mathrm{d} s,
$$

here $n=[\alpha]+1$ and $[\alpha]$ denotes the integer part of $\alpha$, when $a=0, D_{a}^{\alpha} h(t)=D^{\alpha} h(t)$.

Definition 2.3 [14]: For a function $h$ given on the interval $[a, b]$, the Caputo fractional-order derivative of order $\alpha$ of $h$, is defined by

$$
{ }^{c} D_{a}^{\alpha} h(t)=\frac{1}{\Gamma(n-\alpha)} \int_{a}^{t}(t-s)^{n-\alpha-1} h^{(n)}(s) \mathrm{d} s,
$$

where $n=[\alpha]+1$.

Lemma 2.4 [25]: (Schaefer's fixed point theorem). Let $X$ be a Banach space and $F: X \rightarrow X$ be a completely continuous operator. If the set $\mathrm{E}=\{y \in X: y=\lambda F(y), 0<\lambda<1\}$ is bounded, then $F$ has at least a fixed point in $X$.

\section{Existence of Solutions}

Consider the set of functions

$$
\begin{aligned}
& P C(J, R) \\
= & \left\{y(t): J \rightarrow R ; y(t) \in C\left(\left(t_{k}, t_{k+1}\right], R\right), k=0,1, \cdots, m\right. \\
& \text { and there existy }\left(t_{k}^{-}\right) \text {and } y\left(t_{k}^{+}\right), k=1,2, \cdots, m \\
& \text { with } \left.y\left(t_{k}^{-}\right)=y\left(t_{k}\right)\right\} .
\end{aligned}
$$

Definition 3.1: A function $y(t) \in P C(J, R)$ whose $\alpha$-derivative exists on $J$ is said to be a solution of (1.1)-(1.3), if $y$ satisfies the equation

$$
{ }^{c} D^{\alpha} y(t)=f\left(t, y(t), \int_{0}^{t} h(t, r) y(r) \mathrm{d} r\right),
$$

on $J^{\prime}$ and satisfies the conditions

$$
\begin{aligned}
& \left.\Delta y(t)\right|_{t=t_{k}}=I_{k}\left(y\left(t_{k}^{-}\right)\right), k=1,2, \cdots, m, \\
& y(0)+g(y(t))=y_{0}
\end{aligned}
$$

where $J^{\prime}=[0, T] /\left\{t_{1}, t_{2}, \cdots, t_{m}\right\}$.

To prove the existence of solutions to (1.1)-(1.3), we need the following auxiliary lemmas.

Lemma 3.2: Let $\alpha>0$, then the equation

$$
{ }^{c} D^{\alpha} h(t)=0
$$

has solutions

$$
\begin{aligned}
& h(t)=c_{0}+c_{1} t+\cdots+c_{n-1} t^{n-1}, \\
& \left(c_{i} \in R, i=1,2, \cdots, n-1, n=[\alpha]+1\right) .
\end{aligned}
$$

Lemma 3.3: Let $\alpha>0$, then

$$
I^{\alpha c} D^{\alpha} h(t)=h(t)+c_{0}+c_{1} t+\cdots+c_{n-1} t^{n-1},
$$

for some $c_{i} \in R, i=1,2, \cdots, n-1, n=[\alpha]+1$.

As a consequence of Lemma 3.2 and Lemma 3.3, we have the following result

Lemma 3.4: Let $0<\alpha<1$, and let $h: J \rightarrow R$ be continuous. A function $y$ is a solution of the fractional integral equation

$$
y(t)=\left\{\begin{array}{l}
y_{0}-g(y(t))+\frac{1}{\Gamma(\alpha)} \int_{0}^{t}(t-s)^{\alpha-1} h(s) \mathrm{d} s, \\
\text { if } t \in\left[0, t_{1}\right], \\
y_{0}-g(y(t))+\frac{1}{\Gamma(\alpha)} \sum_{i=1}^{k} \int_{t_{i-1}}^{t_{i}}\left(t_{i}-s\right)^{\alpha-1} h(s) \mathrm{d} s \\
+\frac{1}{\Gamma(\alpha)} \int_{t_{k}}^{t}(t-s)^{\alpha-1} h(s) \mathrm{d} s \\
+\sum_{i=1}^{k} I_{i}\left(y\left(t_{i}^{-}\right)\right), \text {if } t \in\left[t_{k}, t_{k+1}\right],(k=1,2, \cdots, m),
\end{array}\right.
$$

if and only if $y(t)$ is a solution of the fractional nonlocal BVP

$$
\begin{gathered}
{ }^{c} D^{\alpha} y(t)=h(t), t \in J^{\prime}, \\
\left.\Delta y(t)\right|_{t=t_{k}}=I_{k}\left(y\left(t_{k}^{-}\right)\right), k=1,2, \cdots, m, \\
y(0)+g(y(t))=y_{0} .
\end{gathered}
$$

Proof Assume $y(t)$ satisfies (3.2)-(3.4). If $t \in\left[0, t_{1}\right]$ then ${ }^{c} D^{\alpha} y(t)=h(t)$. 
Lemma 3.3 implies

$$
y(t)=y_{0}-g(y(t))+\frac{1}{\Gamma(\alpha)} \int_{0}^{t}(t-s)^{\alpha-1} h(s) \mathrm{d} s .
$$

If $t \in\left[t_{1}, t_{2}\right]$, by Lemma 3.3, it follows that

$$
\begin{aligned}
& y(t)=y\left(t_{1}^{+}\right)+\frac{1}{\Gamma(\alpha)} \int_{t_{1}}^{t}(t-s)^{\alpha-1} h(s) \mathrm{d} s \\
& =\left.\Delta y(t)\right|_{t=t_{1}}+y\left(t_{1}^{-}\right)+\frac{1}{\Gamma(\alpha)} \int_{t_{1}}^{t}(t-s)^{\alpha-1} h(s) \mathrm{d} s \\
& =I_{1}\left(y\left(t_{1}^{-}\right)\right)+y_{0}-g(y)+\frac{1}{\Gamma(\alpha)} \int_{0}^{t_{1}}\left(t_{1}-s\right)^{\alpha-1} h(s) \mathrm{d} s \\
& +\frac{1}{\Gamma(\alpha)} \int_{t_{1}}^{t}(t-s)^{\alpha-1} h(s) \mathrm{d} s .
\end{aligned}
$$

If $t \in\left[t_{2}, t_{3}\right]$, then from Lemma 3.3 we get

$$
\begin{aligned}
& y(t)=y\left(t_{2}^{+}\right)+\frac{1}{\Gamma(\alpha)} \int_{t_{2}}^{t}(t-s)^{\alpha-1} h(s) \mathrm{d} s \\
& =\left.\Delta y\right|_{t=t_{2}}+y\left(t_{2}^{-}\right)+\frac{1}{\Gamma(\alpha)} \int_{t_{2}}^{t}(t-s)^{\alpha-1} h(s) \mathrm{d} s \\
& =I_{2}\left(y\left(t_{2}^{-}\right)\right)+I_{1}\left(y\left(t_{1}^{-}\right)\right)+y_{0}-g(y(t)) \\
& +\frac{1}{\Gamma(\alpha)} \int_{0}^{t_{1}}\left(t_{1}-s\right)^{\alpha-1} h(s) \mathrm{d} s \\
& +\frac{1}{\Gamma(\alpha)} \int_{t_{1}}^{t_{2}}\left(t_{2}-s\right)^{\alpha-1} h(s) \mathrm{d} s+\frac{1}{\Gamma(\alpha)} \int_{t_{2}}^{t}(t-s)^{\alpha-1} h(s) \mathrm{d} s .
\end{aligned}
$$

If $t \in\left[t_{k}, t_{k+1}\right]$, then again from $t \in\left[t_{2}, t_{3}\right]$ we have (3.1).

Conversely, assume that $y$ satisfies the impulsive fractional integral equation (3.1). If $t \in\left[0, t_{1}\right]$, then $y(0)+g(y(t))=y_{0}$ and using the fact that ${ }^{c} D^{\alpha}$ is the left inverse of $I^{\alpha}$, we get ${ }^{c} D^{\alpha} y(t)=h(t)$.

If $t \in\left[t_{k}, t_{k+1}\right], k=1,2, \cdots, m$ and using the fact that ${ }^{c} D^{\alpha} C=0$, where $C$ is a constant, we conclude that ${ }^{c} D^{\alpha} y(t)=h(t)$.

Also, we can easily show that

$$
\left.\Delta y\right|_{t=t_{k}}=I_{k}\left(y\left(t_{k}^{-}\right)\right), k=1,2, \cdots, m .
$$

Theorem: Assume that:

$\left(\mathrm{H}_{1}\right)$ There exists a constant $M>0$ such that $|f(t, u, v)| \leq M$ for each $t \in J$ and each $u, v \in R$;

$\left(\mathrm{H}_{2}\right)$ There exists a constant $l_{k}>0$ such that $\left|I_{k}(u)\right| \leq l_{k}$, for each $u \in R$ and $k=1,2, \cdots, m$;

$\left(\mathrm{H}_{3}\right)$ There exists a constant $l>0$ such that $|g(u)| \leq l$, for each $u \in P C(J, R)$, then the problem (1.1)-(1.3) has at least one solution on $J$.

Proof Consider the operator

$F: P C(J, R) \rightarrow P C(J, R)$ defined by

$$
F(y(t))=\left\{\begin{array}{l}
y_{0}-g(y(t))+\frac{1}{\Gamma(\alpha)} \int_{0}^{t}(t-s)^{\alpha-1} f\left(s, y(s), \int_{0}^{s} h(s, r) y(r) \mathrm{d} r\right) \mathrm{d} s, \text { if } t \in\left[0, t_{1}\right], \\
y_{0}-g(y(t))+\frac{1}{\Gamma(\alpha)} \sum_{i=1}^{k} \int_{t_{i-1}}^{t_{i}}\left(t_{i}-s\right)^{\alpha-1} f\left(s, y(s), \int_{0}^{s} h(s, r) y(r) \mathrm{d} r\right) \mathrm{d} s \\
\quad+\frac{1}{\Gamma(\alpha)} \int_{t_{k}}^{t}(t-s)^{\alpha-1} f\left(s, y(s), \int_{0}^{s} h(s, r) y(r) \mathrm{d} r\right) \mathrm{d} s+\sum_{i=1}^{k} I_{i}\left(y\left(t_{i}^{-}\right)\right), \text {if } t \in\left[t_{k}, t_{k+1}\right],(k=1,2, \cdots, m),
\end{array}\right.
$$

Clearly, the fixed points of the operator $F$ are solution of the problem (1.1)-(1.3).

We shall use Schaefer's fixed point theorem to prove that $F$ has a fixed point. The proof will be given in several steps.

Step 1: $F$ is continuous.

Let $\left\{y_{n}(t)\right\}$ be a sequence such that $y_{n} \rightarrow y$ in $P C(J, R)$. Then for each

$$
\begin{aligned}
& t \in J_{0}=\left[0, t_{1}\right],\left|F\left(y_{n}(t)\right)-F(y(t))\right| \\
& \leq \frac{1}{\Gamma(\alpha)} \int_{0}^{t}(t-s)^{\alpha-1}\left|f\left(s, y_{n}(s), \int_{0}^{s} h(s, r) y_{n}(r) \mathrm{d} r\right)-f\left(s, y(s), \int_{0}^{s} h(s, r) y(r) \mathrm{d} r\right)\right| \mathrm{d} s .
\end{aligned}
$$

Since $f$ is continuous function, we have $\left|F\left(y_{n}(t)\right)-F(y(t))\right| \rightarrow 0$, as $n \rightarrow \infty$.

For each $t \in J_{k}=\left[t_{k}, t_{k+1}\right]$,

$$
\begin{aligned}
& \left|F\left(y_{n}(t)\right)-F(y(t))\right| \leq \frac{1}{\Gamma(\alpha)} \sum_{i=1}^{k} \int_{t_{i-1}}^{t_{i}}\left(t_{i}-s\right)^{\alpha-1} \times\left|f\left(s, y_{n}(s), \int_{0}^{s} h(s, r) y_{n}(r) \mathrm{d} r\right)-f\left(s, y(s), \int_{0}^{s} h(s, r) y(r) \mathrm{d} r\right)\right| \mathrm{d} s \\
& +\frac{1}{\Gamma(\alpha)} \int_{t_{k}}^{t}\left(t_{i}-s\right)^{\alpha-1} \times\left|f\left(s, y_{n}(s), \int_{0}^{s} h(s, r) y_{n}(r) \mathrm{d} r\right)-f\left(s, y(s), \int_{0}^{s} h(s, r) y(r) \mathrm{d} r\right)\right| \mathrm{d} s+\sum_{i=1}^{k}\left|I_{i}\left(y_{n}\left(t_{i}^{-}\right)\right)-I_{i}\left(y\left(t_{i}^{-}\right)\right)\right| .
\end{aligned}
$$


Since $f$ and $I_{i}, i=1,2, \cdots, m$ are continuous functions, we have $\left|F\left(y_{n}(t)\right)-F(y(t))\right| \rightarrow 0$, as $n \rightarrow \infty$.

Therefore, $F$ is continuous.

Step 2: $F$ maps bounded sets into bounded sets in $P C(J, R)$.

Indeed, it is enough to show that for any $\eta^{*}>0$, there exists a positive constant $\ell$ such that for each $y \in B_{\eta^{*}}=\left\{y \in P C(J, R):\|y\|_{\infty} \leq \eta^{*}\right\}$, we have $\|F(y)\| \leq \ell$. By $\left(\mathrm{H}_{1}\right),\left(\mathrm{H}_{2}\right)$ and $\left(\mathrm{H}_{3}\right)$, for each $t \in\left[0, t_{1}\right]$, we have

$$
\begin{aligned}
& |F(y(t))| \leq\left|y_{0}\right|+|g(y(t))|+\frac{1}{\Gamma(\alpha)} \int_{0}^{t}(t-s)^{\alpha-1} \\
& \qquad\left|f\left(s, y(s), \int_{0}^{s} h(s, r) y(r) \mathrm{d} r\right)\right| \mathrm{d} s \\
& \leq\left|y_{0}\right|+l+\frac{M}{\Gamma(\alpha)} \int_{0}^{t}(t-s)^{\alpha-1} \mathrm{~d} s \leq\left|y_{0}\right|+l+\frac{M}{\Gamma(\alpha+1)} t^{\alpha} \\
& \leq\left|y_{0}\right|+l+\frac{M T^{\alpha}}{\Gamma(\alpha+1)} .
\end{aligned}
$$

For $t \in\left[t_{k}, t_{k+1}\right],(k=1,2, \cdots, m)$, we have

$$
\begin{aligned}
& |F(y(t))| \leq\left|y_{0}\right|+|g(y(t))|+\frac{1}{\Gamma(\alpha)} \sum_{i=1}^{k} \int_{t_{i-1}}^{t_{i}}\left(t_{i}-s\right)^{\alpha-1}\left|f\left(s, y(s), \int_{0}^{s} h(s, r) y(r) \mathrm{d} r\right)\right| \mathrm{d} s \\
& +\frac{1}{\Gamma(\alpha)} \int_{t_{k}}^{t}(t-s)^{\alpha-1}\left|f\left(s, y(s), \int_{0}^{s} h(s, r) y(r) \mathrm{d} r\right)\right| \mathrm{d} s+\sum_{i=1}^{k}\left|I_{i}\left(y\left(t_{i}^{-}\right)\right)\right| \\
& \leq\left|y_{0}\right|+l+\frac{M}{\Gamma(\alpha+1)} \sum_{i=1}^{k} t_{i}^{\alpha}+\frac{M}{\Gamma(\alpha+1)} t^{\alpha}+\sum_{i=1}^{k} l_{i} \leq\left|y_{0}\right|+l+\frac{(k+1) M T^{\alpha}}{\Gamma(\alpha+1)}+\sum_{i=1}^{k} l_{i} .
\end{aligned}
$$

Let

$$
\begin{aligned}
& \ell=\max \left\{\left|y_{0}\right|+l+\frac{M T^{\alpha}}{\Gamma(\alpha+1)},\left|y_{0}\right|+l+\frac{(k+1) M T^{\alpha}}{\Gamma(\alpha+1)}+\sum_{i=1}^{k} l_{i}\right\}, \\
& k=1,2, \cdots, m, \\
& \text { then }\|F(y(t))\| \leq \ell .
\end{aligned}
$$

$$
\begin{aligned}
& \left|F\left(y\left(\tau_{2}\right)\right)-F\left(y\left(\tau_{1}\right)\right)\right| \\
& =\frac{1}{\Gamma(\alpha)}\left|\int_{0}^{\tau_{2}}\left(\tau_{2}-s\right)^{\alpha-1} f\left(s, y(s), \int_{0}^{s} h(s, r) y(r) \mathrm{d} r\right) \mathrm{d} s-\int_{0}^{\tau_{1}}\left(\tau_{1}-s\right)^{\alpha-1} f\left(s, y(s), \int_{0}^{s} h(s, r) y(r) \mathrm{d} r\right) \mathrm{d} s\right| \\
& \leq \frac{1}{\Gamma(\alpha)} \int_{0}^{\tau_{2}}\left|\left(\tau_{2}-s\right)^{\alpha-1}-\left(\tau_{1}-s\right)^{\alpha-1}\right| \times\left|f\left(s, y(s), \int_{0}^{s} h(s, r) y(r) \mathrm{d} r\right)\right| \mathrm{d} s \\
& +\frac{1}{\Gamma(\alpha)} \int_{\tau_{1}}^{\tau_{2}}\left|\left(\tau_{2}-s\right)^{\alpha-1}\right| \times\left|f\left(s, y(s), \int_{0}^{s} h(s, r) y(r) \mathrm{d} r\right)\right| \mathrm{d} s \\
& \leq \frac{M}{\Gamma(\alpha)} \int_{0}^{\tau_{2}}\left|\left(\tau_{2}-s\right)^{\alpha-1}-\left(\tau_{1}-s\right)^{\alpha-1}\right| \mathrm{d} s+\frac{M}{\Gamma(\alpha)} \int_{\tau_{1}}^{\tau_{2}}\left|\left(\tau_{2}-s\right)^{\alpha-1}\right| \mathrm{d} s \leq \frac{M}{\Gamma(\alpha+1)}\left|2\left(\tau_{2}-\tau_{1}\right)^{\alpha}+\tau_{2}^{\alpha}-\tau_{1}^{\alpha}\right| \mathrm{d} s .
\end{aligned}
$$

Step 3: $F$ maps bounded sets into equicontinuous sets of $P C(J, R)$.

Let $\tau_{1}, \tau_{2} \in J, \tau_{1}<\tau_{2}, \quad B_{\eta^{*}}$ be a bounded set of $P C(J, R)$ as in Step 2, and let $y \in B_{\eta^{*}}$. For $\tau_{1}, \tau_{2} \in\left[0, t_{1}\right]$, we have

For $\tau_{1}, \tau_{2} \in\left[t_{k}, t_{k+1}\right],(k=1,2, \cdots, m)$, we have

$$
\begin{aligned}
& \left|F\left(y\left(\tau_{2}\right)\right)-F\left(y\left(\tau_{1}\right)\right)\right| \leq+\sum_{0<t_{k}<\tau_{2}-\tau_{1}}\left|I_{k}\left(y\left(t_{k}^{-}\right)\right)\right| \\
& +\frac{1}{\Gamma(\alpha)}\left|\int_{0}^{\tau_{2}}\left(\tau_{2}-s\right)^{\alpha-1} f\left(s, y(s), \int_{0}^{s} h(s, r) y(r) \mathrm{d} r\right) \mathrm{d} s-\int_{0}^{\tau_{1}}\left(\tau_{1}-s\right)^{\alpha-1} f\left(s, y(s), \int_{0}^{s} h(s, r) y(r) \mathrm{d} r\right) \mathrm{d} s\right| \\
& \leq \sum_{0<t_{k}<\tau_{2}-\tau_{1}}\left|I_{k}\left(y\left(t_{k}^{-}\right)\right)\right|+\frac{1}{\Gamma(\alpha)} \int_{0}^{\tau_{2}}\left|\left(\tau_{2}-s\right)^{\alpha-1}-\left(\tau_{1}-s\right)^{\alpha-1}\right| \times\left|f\left(s, y(s), \int_{0}^{s} h(s, r) y(r) \mathrm{d} r\right)\right| \mathrm{d} s \\
& +\frac{1}{\Gamma(\alpha)} \int_{\tau_{1}}^{\tau_{2}}\left|\left(\tau_{2}-s\right)^{\alpha-1}\right| \times\left|f\left(s, y(s), \int_{0}^{s} h(s, r) y(r) \mathrm{d} r\right)\right| \mathrm{d} s \\
& \leq \sum_{0<t_{k}<\tau_{2}-\tau_{1}}\left|I_{k}\left(y\left(t_{k}^{-}\right)\right)\right|+\frac{M}{\Gamma(\alpha)} \int_{0}^{\tau_{2}}\left|\left(\tau_{2}-s\right)^{\alpha-1}-\left(\tau_{1}-s\right)^{\alpha-1}\right| \mathrm{d} s+\frac{M}{\Gamma(\alpha)} \int_{\tau_{1}}^{\tau_{2}}\left|\left(\tau_{2}-s\right)^{\alpha-1}\right| \mathrm{d} s \\
& \leq \sum_{0<\tau_{k}<\tau_{2}-\tau_{1}}\left|I_{k}\left(y\left(t_{k}^{-}\right)\right)\right|+\frac{M}{\Gamma(\alpha+1)}\left|2\left(\tau_{2}-\tau_{1}\right)^{\alpha}+\tau_{2}^{\alpha}-\tau_{1}^{\alpha}\right| \mathrm{d} s .
\end{aligned}
$$


As $\tau_{1} \rightarrow \tau_{2}$, the right-hand side of the above inequality tends to zero. As a consequence of Steps 1 to 3 together with the Arzel'a-Ascoli theorem, we can conclude that $F: P C(J, R) \rightarrow P C(J, R)$ is completely continuous.

As a consequence of Lemma 2.4 (Schaefer's fixed point theorem), we deduce that $F$ has a fixed point which is a solution of the problem (1.1)-(1.3).

\section{Acknowledgements}

This work was supported by the natural science foundation of Hunan Province (13JJ6068, 12JJ9001), Hunan provincial science and technology department of science and tech-neology project (2012SK3117), Science foundation of Hengyang normal university of China (No. 12B35) and Construct program of the key discipline in Hunan Province.

\section{REFERENCES}

[1] J. A. Tenreiro Machado, V. Kiryakova and F. Mainardi, "Recent History of Fractional Calculus," Communications in Nonlinear Science and Numerical Simulation, Vol. 16, No. 3, 2011, pp. 1140-1153.

[2] A. A. Kilbas, H. M. Srivastava and J. J. Trujillo, "Theory and Applications of Fractional Differential Equations," North-Holland Mathematics Studies, Vol. 204, 2006. doi:10.1016/S0304-0208(06)80001-0

[3] K. Diethelm, “The Analysis of Fractional Differential Equations,” Springer-Verlag, Berlin, Heidelberg, 2010. doi:10.1007/978-3-642-14574-2

[4] K. S. Miller and B. Ross, "An Introduction to the Fractional Calculus and Fractional Differential Equations,” John Wiley, New York, 1993.

[5] I. Podlubny, "Fractional Differential Equations," Academic Press, San Diego, New York, London, 1999.

[6] S. G. Samko, A. A. Kilbas and O. I. Marichev, "Fractional Integral and Derivatives," Gordon and Breach Science Publisher, London, 1993.

[7] J. Sabatier, O. P. Agrawal and J. A. Tenreiro Machado, "Advances in Fractional Calculus: Theoretical Developments and Applications in Physics and Engineering," Springer, Berlin, 2007. doi:10.1007/978-1-4020-6042-7

[8] V. Lakshmikantham, S. Leela and J. Vasundhara Devi, "Theory of Fractional Dynamic Systems," Cambridge Academic Publishers, Cambridge, 2009.

[9] S. Zhang, "Positive Solutions for Boundary-Value Problems of Nonlinear Fractional Differential Equations," Electronic Journal of Qualitative Theory of Differential Equations, Vol. 36, 2006, pp. 1-12.

[10] B. Ahmad and J. J. Nieto, "Existence of Solution for Non-Local Boundary Value Problems of Higher-Order Nonlinear Fractional Differential Equations,” Abstract and Applied Analysis, Vol. 2009, 2009, pp. 1-9. doi: $10.1155 / 2009 / 494720$

[11] A. A. Kilbas and S. A. Marzan, "Nonlinear Differential
Equations with the Caputo Fractional Derivative in the Space of Continuously Differentiable Functions," Differential Equations, Vol. 41, No. 1, 2005, pp. 84-89. doi:10.1007/s10625-005-0137-y

[12] V. D. Milman and A. D. Myshkis, "On the Stability of Motion in the Presence of Impulses (Russian)," Siberial Mathematical Journal, Vol. 1, No. 2, 1960, pp. 233-237.

[13] A. M. Samoilenko and N. A. Perestyuk, "Differential Equations with Impulses,” Viska Scola, Kiev, 1987 (in Russian).

[14] V. Lakshmikantham, D. D. Baino and P. S. Simeonov, “Theory of Impulsive Differential Equations," World Scientific Publishing Corporation, Singapore City, 1989. doi:10.1142/0906

[15] D. D. Baino and P. S. Simeonov, "Systems with Impulsive Effects,” Horwood, Chichister, 1989.

[16] D. D. Baino and P. S. Simeonov, "Impulsive Differential Equations: Periodic Solutions and Its applications," Longman Scientific and Technical Group, England, 1993.

[17] D. D. Baino and V. C. Covachev, "Impulsive Differential Equations with a Small Perturbations,” World Scientific, New Jersey, 1994. doi:10.1142/2058

[18] M. Benchohra, J. Henderson and S. K. Ntonyas, "Impulsive Differential Equations and Inclusions,” Hindawi Publishing Corporation, New York, 2006. doi:10.1155/9789775945501

[19] R. P. Agarwal, M. Benchohra and B. A. Salimani, "Existence Results for Differential Equations with Fractional Order and Impulses," Memoir on Differential Equations and Mathematical Physics, Vol. 44, 2008, pp. 1-21.

[20] M. Benchohra and B. A. Salimani, "Existence and Uniqueness of Solutions to Impulsive Fractional Differential Equations,” Electronic Journal of Differential Equations, Vol. 2009, No. 10, 2009, pp. 1-11.

[21] M. Fecken, Y. Zhong and J. Wang, "On the Concept and existence of Solutions for Impulsive Fractional Differential Equations," Communications in Non-Linear Science and numerical Simulation, Vol. 17, No. 7, 2012, pp. 3050-3060. doi:10.1016/j.cnsns.2011.11.017

[22] L. Byszewski and V. Lakshmikantham, "Theorem about the Existence and Uniqueness of a Solution of a Nonlocal Abstract Cauchy Problem in a Banach Space," Journal of Applied Analysis, Vol. 40, 1991, pp. 11-19. doi:10.1080/00036819008839989

[23] L. Byszewski, “Theorems about Existence and Uniqueness of Solutions of a Semilinear Evolution Nonlocal Cauchy Problem,” Journal of Mathematical Analysis and Applications, Vol. 162, No. 2, 1991, pp. 494-505. doi:10.1016/0022-247X(91)90164-U

[24] L. Byszewski, "Existence and Uniqueness of Mild and Classical Solutions of Semilinear Functional-Differential Evolution Nonlocal Cauchy Problem,” Selected Problems of Mathematics, 50th Anniversary Cracow University of Technology, No. 6, Cracow University of Technology, Krakow, 1995, pp. 25-33.

[25] J. X. Sun, "Nonlinear Functional Analysis and Its Application,” Science Press, Beijing, 2008. 